\title{
LV-OP-1-1
}

\section{Elderly living liver donors in Korea}

\author{
Jong Man KIM ${ }^{* 1}$, Nam-Joon $\mathrm{Yl}^{2}$, Dong Jin $\mathrm{JOO}^{3}$, Young Kyoung YOO${ }^{4}$, Shin $\mathrm{HWANG}^{5}$, \\ Je Ho RYU ${ }^{6}$, Doo Jin KIM7', Hee Chul YU ${ }^{8}$, Yang Won NA', Myoung Soo KIM ${ }^{3}$ \\ 'Department of Surgery, Samsung Medical Center, Sungkyunkwan University School of Medicine, Seoul, Korea \\ ${ }^{2}$ Department of Surgery, Seoul National University College of Medicine, Seoul, Korea \\ ${ }^{3}$ Department of Surgery, Yonsei University College of Medicine, Seoul, Korea \\ ${ }^{4}$ Department of Surgery, College of Medicine, The Catholic University of Korea, Seoul, Korea \\ ${ }^{5}$ Department of Surgery, Asan Medical Center, University of Ulsan College of Medicine, Seoul, Korea \\ ${ }^{6}$ Department of Surgery, Pusan National University College of Medicine, Yangsan, Korea \\ 7Department of Surgery, Gachon University Gil Medical Center, Gachon University College of Medicine, Incheon, Korea \\ ${ }^{8}$ Department of Surgery, Jeonbuk National University Medical School, Jeonju, Korea \\ ${ }^{9}$ Department of Surgery, Ulsan University Hospital, University of Ulsan College of Medicine, Ulsan, Korea
}

Introduction: Old donors have gradually been used as an alternative living liver donor to alleviate the organ shortage or avoid offspring donation. The aim of the present study is to determine the impact of elderly donors more than 60 years in living donor liver transplantation (LDLT) on donor safety and recipient outcomes compared with donors from fifty to fifty-nine years.

Methods: We retrospectively identified 209 patients at nine centers from 2005 to 2017 in Korea.

Results: Sixty group represented $10.0 \%(\mathrm{n}=21)$ of the patient donors. The incidence of male donor in the sixty group was higher than in the fifty group $(61.9 \%$ vs. $37.8 \% ; p=0.039)$. Postoperative complications were more common in the sixty group. There was no in-hospital mortality and no mortality was reported during the observation period. There were no statistically significant differences in operation time, blood loss, intraoperative and postoperative transfusion rate, postoperative total bilirubin, and hospitalization between the two groups. In recipient operation, median blood loss, transfusion rates during operation, and postoperative bleeding control operations were more than in the fifty group. Postoperative total bilirubin and hospitalization in the sixty group were higher and longer in the fifty group. Cumulative patient survival rate in the fifty group was better than in the sixty group $(p=0.011)$. Sixty group was predisposing factor for recipient death in multivariate analysis.

Conclusions: Present study suggests that highly selected elderly living donors ( $\geq 60$ years) can safely donate, but their recipient outcomes are worse compared with the fifty group. 